\title{
Revisión a la seguridad alimentaria en el Ecuador
}

\author{
Food security in Ecuador
}

DOI: $10.46932 / \operatorname{sfjdv2n2-157}$

Received in: March 1st, 2021

Accepted in: May 30th, 2021

Ing. Com. Guido Homero Poveda Burgos

Magister en Administración y Dirección de Empresas

Docente de la Universidad de Guayaquil

E-mail: guido.povedabu@ug.edu.ec

Ing. Com. Vicente Bernabé Salazar Soledispa

Magister en Administración de Empresas mención en Negocios Internacionales

Docente de la Universidad de Guayaquil

E-mail: salazarsvb@ug.edu.ec

Ing. Com. Pedro Alexander Avilés Almeida

Magister en Educación Superior

Docente de la Universidad de Guayaquil

E-mail: pedro.avilesal@ug.edu.ec

Ing. Com. Jean Steve Carrera López

Magister en Administración de Empresas Mención Negocios Internacionales

Docente de la Universidad de Guayaquil

E-mail: jean.carreralo@ug.edu.ec

Ing. Com. Gabriel de Jesús Neira Vera

Magister en Administración y Dirección de Empresas

Docente de la Universidad de Guayaquil

E-mail: gabriel.neirav@ug.edu.ec

\section{RESUMEN}

En el presente trabajo se analizará sobre la seguridad alimentaria, en su evolución que se ha dado desde los años 70 esto se dio basándose en la producción y disponibilidad alimentaria a nivel global y nacional, en los años 80 se le añadió una idea de acceso, tanto económico como físico y en la década del 90 se llegó al concepto actual que incorpora la inocuidad, las preferencias culturales y se reafirma la Seguridad Alimentaria como derecho humano.

La Seguridad alimentaria está estructurada en función de cuatro componentes básicos como es la disponibilidad, estabilidad, acceso a los alimentos, consumos de los alimentos y la utilización biológica de los alimentos en cada uno se especifica su concepto principal, también se menciona el derecho a la alimentación en el Ecuador así como los organismos, planes y objetivos el cual tiene que cumplir Ecuador si es que se desea disminuir la pobreza y la desnutrición, como también las causas de la inseguridad alimentaria, uno de los objetivos principal es el análisis de cómo se encuentra actualmente la Seguridad Alimentaria del Ecuador por medio de los cuatro componentes en el que está estructurada la Seguridad Alimentaria y por medio de datos estadísticos actualizados y así poder llegar a una conclusión de cómo se encuentra la Seguridad Alimentaria en el Ecuador. 
Palabras Clave: Seguridad Alimentaria, evolución, acceso a los alimentos, Derecho humano, componentes.

\begin{abstract}
This paper analyzes the evolution of food security since the 1970s, based on global and national food production and availability. In the 1980s, the idea of access, both economic and physical, was added, and in the 1990s the current concept was arrived at, which incorporates food security, cultural preferences and reaffirms food security as a human right.

Food security is structured in terms of four basic components such as availability, stability, access to food, food consumption and biological utilization of food, each of which specifies its main concept. One of the main objectives is the analysis of the current state of food security in Ecuador through the four components in which food security is structured and through updated statistical data to reach a conclusion on the state of food security in Ecuador.
\end{abstract}

Key words: food security, evolution, access to food, human right, components.

\title{
1 INTRODUCCIÓN
}

Según la FAO, seguridad alimentaria es que "todas las personas tienen en todo momento acceso físico y económico a suficientes alimentos inocuos y nutritivos para satisfacer sus necesidades alimentarias", para lo cual tiene que haber disponibilidad de los alimentos, capacidad para adquirirlos, estabilidad en la oferta, buena calidad e inocuidad; siendo el productor el primer eslabón en producir alimento saludable; el sector industrial transformador tiene que utilizar materia prima adecuada aplicando las normativas establecidas; una empresa tiene con frecuencia que valorar si sus productos, procesos e instalaciones o los de sus proveedores responden a las especificaciones y estándares que le exige el mercado o la legislación vigente, para ello es de gran utilidad las auditorías internas que obligadamente debe disponer la propia empresa. La amenaza al hambre es constante; por lo que se requiere implantar programas de seguridad alimentaria para proteger la población de menor capacidad en adquirir alimento la que es más vulnerable a brotes epidémicos.

La facultad suprema del hombre es el derecho a la vida y no hay vida si no dispone de alimento, para lo cual se deben aplicar métodos de medición de seguridad alimentaria como son los índices de disponibilidad de la oferta y demanda, de accesibilidad de la canasta familiar para evitar desnutrición.

El control de la calidad e inocuidad de los alimentos es responsabilidad de todos los involucrados en la cadena alimentaria, desde los productos primarios agricultores y ganaderos, a los procesadores, envasadores, transportadores, almacenadores, punto de ventas y por último el consumidor que exige que las medidas concernientes a la vigilancia y control de la seguridad alimentaria se cumplan, reflejándose en la aceptación o rechazo del producto ofertado.

Las administraciones de los diferentes centros de producción y comercialización, tanto nacional como local, tienen que aplicar diariamente auditorias para verificar la calidad e inocuidad de los alimentos; los puntos de ventas tienen en sus manos la responsabilidad directa ante el consumidor al 
ofertar productos alimenticios que cumplan con todas las normativas establecidas que garantizan su naturaleza y que son inofensivos a la salud.

Es oportuno señalar que la inocuidad de los alimentos no es negociable, siendo la etiqueta uno de los medios de control en la que están impreso las características de cada alimento. (Sigifredo Briones Mejía, 2008)

\section{SEGURIDAD ALIMENTARIA EN ECUADOR}

La seguridad alimentaria surge en la década de los años 70, basado en la producción y disponibilidad alimentaria a nivel global y nacional. En los años 80, se añadió la idea del acceso, tanto económico como físico. Y en la década del 90, se llegó al concepto actual que incorpora la inocuidad y las preferencias culturales, y se reafirma la Seguridad Alimentaria como derecho humano.

Estos factores dieron origen a una nueva concepción de seguridad alimentaria definida en la Cumbre Mundial sobre la Alimentación en 1996. El concepto establecido en esta cumbre y utilizado hasta la actualidad plantea que "existe seguridad alimentaria cuando todas las personas tienen en todo momento acceso físico y económico a suficientes alimentos inocuos y nutritivos para satisfacer sus necesidades alimenticias y sus preferencias en cuanto a los alimentos a fin de llevar una vida activa y sana. (FAO, 1996)

Es la posibilidad real de la población de satisfacer sus necesidades alimentarias mediante el acceso permanente a una oferta de alimentos, derivados de la producción agropecuaria interna como de la existente en el mercado mundial.

El concepto sigue evolucionando y ha pasado desde una visión macro de la seguridad alimentaria a un análisis micro de la vulnerabilidad en función de las "titulaciones o derechos" de las poblaciones y familias.

También, es denominado Soberanía Alimentaria, la cual busca garantizar un acceso efectivo y permanente de alimentos propicios para el desarrollo humano; hacer visible el derecho de los pueblos a definir sus propias políticas en materia de alimentación y nutrición, proteger la producción nacional en el mercado doméstico, en un ambiente de igualdad, paz, sin pobreza y de desarrollo sostenible acompañado de políticas comerciales leales.

La seguridad alimentaria se estructura en función de cuatro componentes básicos, los cuales deben ser analizados conjuntamente con la finalidad de lograr una visión más holística sobre la Seguridad Alimentaria.

\subsection{DISPONIBILIDAD}


a nivel local o nacional tiene en cuenta la producción, las importaciones, almacenamiento y ayuda alimentaria. Estimadas por las pérdidas postcosecha y las exportaciones de alimentos.

\subsection{ESTABILIDAD}

es el solventar las condiciones de Inseguridad Alimentaria transitoria de carácter cíclico o estacional constantemente vinculada con campañas agrícolas debido a la ausencia de alimentos y falta de acceso a recursos de las poblaciones asalariadas en ciertos momentos del año.

Este componente tiene un rol importante para la existencia de infraestructura de almacenamiento a nivel nacional, de la misma manera con la posibilidad de contar con recursos alimenticios.

\subsection{ACCESO A LOS ALIMENTOS}

toma en cuenta el acceso de la gente a los recursos adecuados y el control sobre ello para adquirir, producir alimentos apropiados y generar una alimentación nutritiva.

La falta de acceso y de control son frecuentemente la causa de la Inseguridad Alimentaria y su origen puede ser físico, cuando los alimentos no están disponibles en cantidad suficiente en los lugares donde se necesita consumirlos (poblaciones apartadas), también cuando hay escasez económica, carencia de dinero de las personas para alimentarse con regularidad, calidad y dignidad.

Este aspecto es fundamental, puesto a que, en la mayoría de los casos, y sobre todo en la región de Latinoamérica, la inseguridad alimentaria no se debe a la falta de alimentos sino a los elevados índices de pobrezas que no permiten adquirir los que están disponibles.

\subsection{CONSUMO DE LOS ALIMENTOS}

es la capacidad de la población para resolver problemas debido a la clasificación, almacenamiento, preparación, distribución y consumo de los alimentos. La familia está relacionada a las costumbres, educación, practicas, y a la alimentación e información específica sobre la calidad de los alimentos y nutrición de cada individuo.

También tiene en cuenta aspectos como la inocuidad de los alimentos (food safety), la dignidad de las personas, las condiciones higiénicas de los hogares y la distribución con equidad dentro del hogar.

La evaluación de consumo de alimentos es el interés de la salud pública, es fundamental para orientar sobre la seguridad alimentaria. Es también, fundamental ya que nos permite evitar peligros potenciales (contaminantes, químicos, biológicas, etc.).

\subsection{UTILIZACIÓN BIOLÓGICA DE LOS ALIMENTOS}

está relacionada con el estado nutricional como resultado del uso individual de los alimentos 
(ingestión, absorción y la utilización). La inadecuada utilización biológica puede tener como consecuencia la desnutrición. La educación orienta a la población para mantener el estado de salud por medio de capacitaciones sobre la prevención y los tratamientos de dichas enfermedades. (FAO, 2009)

Existen diversas variables e indicadores que permiten seguir la evolución del nivel de seguridad alimentaria de determinados grupos de población. Dada las dimensiones de la inseguridad alimentaria (crónica, temporal o transitoria) es muy difícil medirla con un solo indicador, para captarla en sus múltiples matrices se necesitan distintos indicadores.

Existen causas de forma individual o en conjunto por la cual se suscita la inseguridad alimentaria, estas pueden ser: la escasez del agua, degradación de los suelos, la contaminación atmosférica, el cambio climático, explosión demográfica, problemas de gobernanza, etc.

\section{EL DERECHO A LA ALIMENTACIÓN EN ECUADOR}

Ecuador reconoce el derecho a la alimentación en su Constitución Política vigente desde el año 2008, como un derecho independiente aplicable a todas las personas.

El artículo 13 establece que las personas y colectividades tienen derecho al acceso seguro y permanente a alimentos sanos, suficientes y nutritivos; preferentemente producidos a nivel local y en correspondencia con sus diversas identidades y tradiciones culturales (Asamblea Nacional, 2008)

La ley orgánica del régimen de Soberanía Alimentaria señala en su artículo 1.- Finalidad. - Esta ley tiene por objeto establecer los mecanismos mediante las cuales el Estado cumpla con su obligación y objetivo estratégico de garantizar a las personas, comunidades y pueblos la autosuficiencia de alimentos sanos, nutritivos y culturalmente apropiados de forma permanente (Ley Orgánica del Régimen de la Soberanía Alimentaria, 2009)

Los 17 Objetivos de Desarrollo Sostenible (ODS) de la Agenda 2030 para el Desarrollo Sostenible aprobada por los dirigentes mundiales en septiembre de 2015 en una cumbre histórica de las Naciones Unidas entraron en vigor oficialmente el 1 de enero de 2016. Con estos nuevos Objetivos de aplicación universal, en los próximos 15 años los países intensificarán los esfuerzos para poner fin a la pobreza en todas sus formas, reducir la desigualdad y luchar contra el cambio climático garantizando, al mismo tiempo, que nadie se quede atrás (Plataforma CELAC, 2015)

Los ODS aprovechan el éxito de los Objetivos de Desarrollo del Milenio (ODM) en el cual Ecuador cumplió con los 8 Objetivos de Desarrollo del Milenio (ODM) y las 21 metas, orientadas a superar la pobreza y mejorar las condiciones de vida en todo el mundo, de igual forma pretende cumplir con los Objetivos de Desarrollo del Milenio para el año 2030.

En enero del 2015 los 33 países de la región, agrupados en la Comunidad de Estados 
Latinoamericanos y Caribeños (CELAC), Aprobaron El Plan para la seguridad alimentaria, la nutrición y erradicación del hambre de la CELAC 2025, elaborado con el apoyo de FAO, CEPAL y ALADI.

Para avanzar hacia la erradicación del hambre, la pobreza y la malnutrición, y garantizar la seguridad alimentaria y nutricional, el Plan CELAC se basa en cuatro pilares que buscan dar respuesta al objetivo de crear una América Latina y el Caribe libre de hambre mediante áreas de acción concretas que, al mismo tiempo, respetan la diversidad de proyectos políticos y sociales propios de cada uno de los países de América Latina y el Caribe (Plataforma CELAC, 2015)

Un tema de importancia que se encuentra a la par de la seguridad alimentaria es la escasez de recursos y el comportamiento humano y empresarial al respecto en términos de responsabilidad social. Elizabeth, R. I. M. (2021) indica:

En la década de los 60 's nace un proceso de concientización acerca de la problemática que se tiene entre las empresas y la escasez de recursos, valores morales y éticos, así como de la economía de las poblaciones, derivando en el concepto de Responsabilidad Empresarial, el cual ha revolucionado $\mathrm{y}$ ha tomado mucha importancia en los últimos años especialmente para estos consumidores (p. 2).

\section{Datos de la Seguridad Alimentaria en Ecuador}

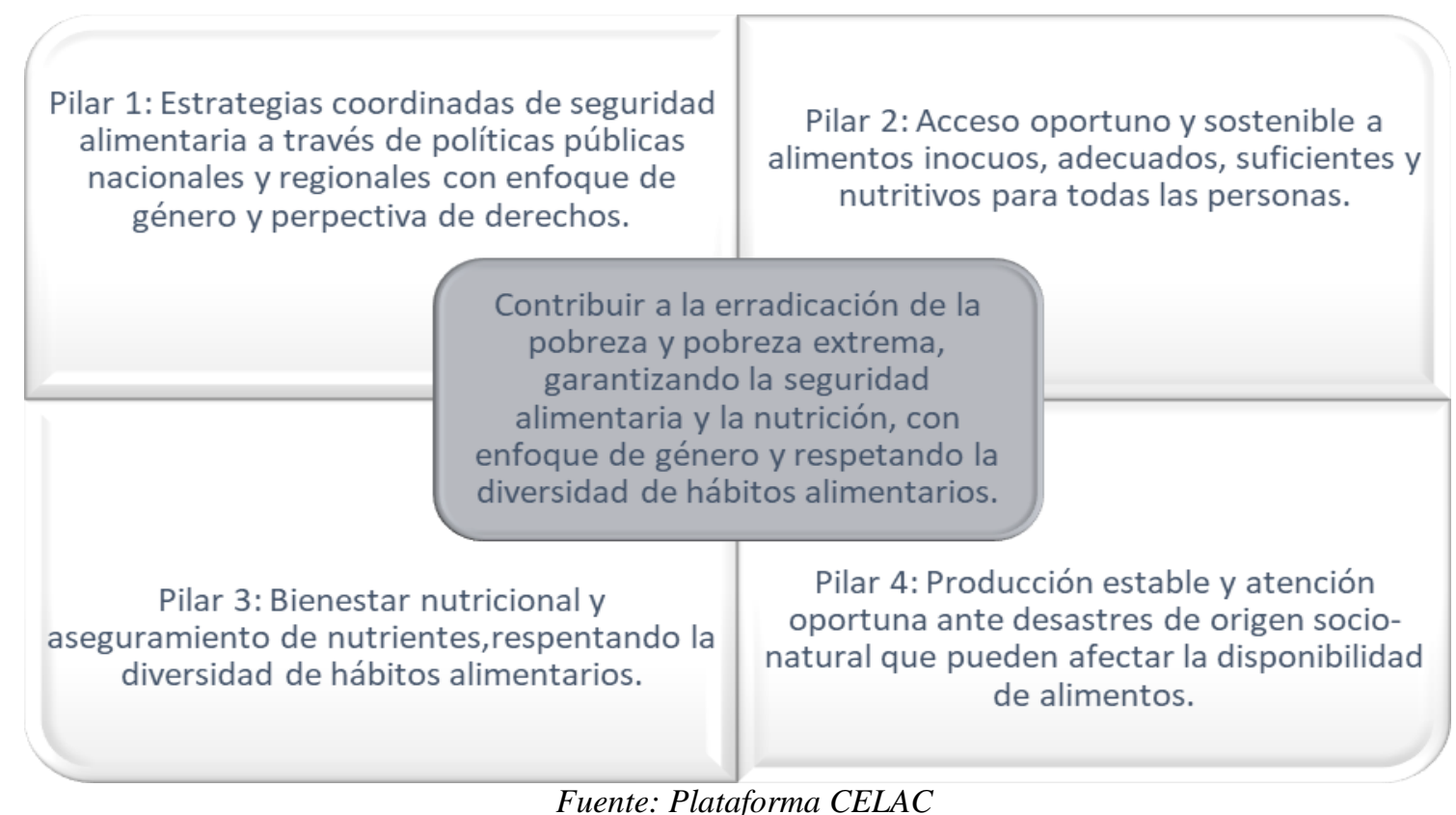

La disponibilidad es el primer indicador de la seguridad alimentaria ya mencionada anteriormente el cual corresponde a la provisión, suministro o existencia de alimentos, es decir aborda lo relacionado con la "oferta", la producción de alimentos, la infraestructura productiva, los insumos y cadenas.

El Producto Interno Bruto (PIB) agroalimentario registró un aumento del 3.5\% en 2016, con lo 
que superó el crecimiento registrado en el PIB nacional, el cual se ubicó en 2.3\% anual.

El avance presentado en el PIB agroalimentario, donde se integran los sectores agroindustrial y primario, fue impulsado por un aumento en la producción primaria de $4.1 \%$ anual, en este segmento la agricultura registró un crecimiento de $5.8 \%$; la ganadería, $2.1 \%$, y la pesca, $0.6 \%$, lo que refleja la potencialidad y las historias de éxito que se generan en el campo nacional.

El Acceso es la forma a través de la cual las personas pueden física y económicamente obtener los alimentos, sea a través del trueque; el autoconsumo; el acceso a los mercados; el aumento del ingreso disponible mediante programas e instrumentos de protección social; o ayuda alimentaria directa.

La subnutrición es parte del acceso y esta ocurre cuando Existe inseguridad alimentaria cuando las personas no tienen acceso físico, social o económico suficiente a alimentos, Subnutrición es el término usado para describir la situación de aquellas personas cuya ingesta de energía alimentaria es por debajo del mínimo requerido para llevar una vida activa.

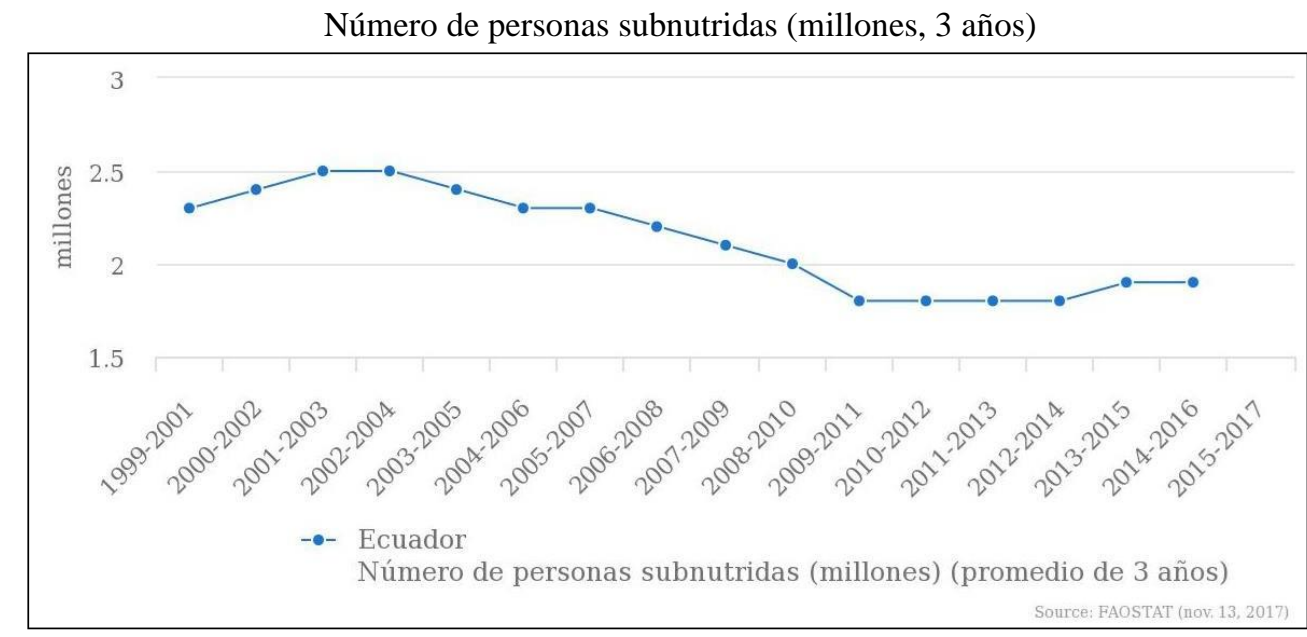

Prevalencia de la subalimentación \% 3 años




En la ilustración se puede observar que el número de personas subnutridas, en el año 2016 han disminuido en comparación de los periodos 2001-2004 y en la ilustración 3 también podemos observar como en los últimos años ha tenido una decreciente en la subalimentación

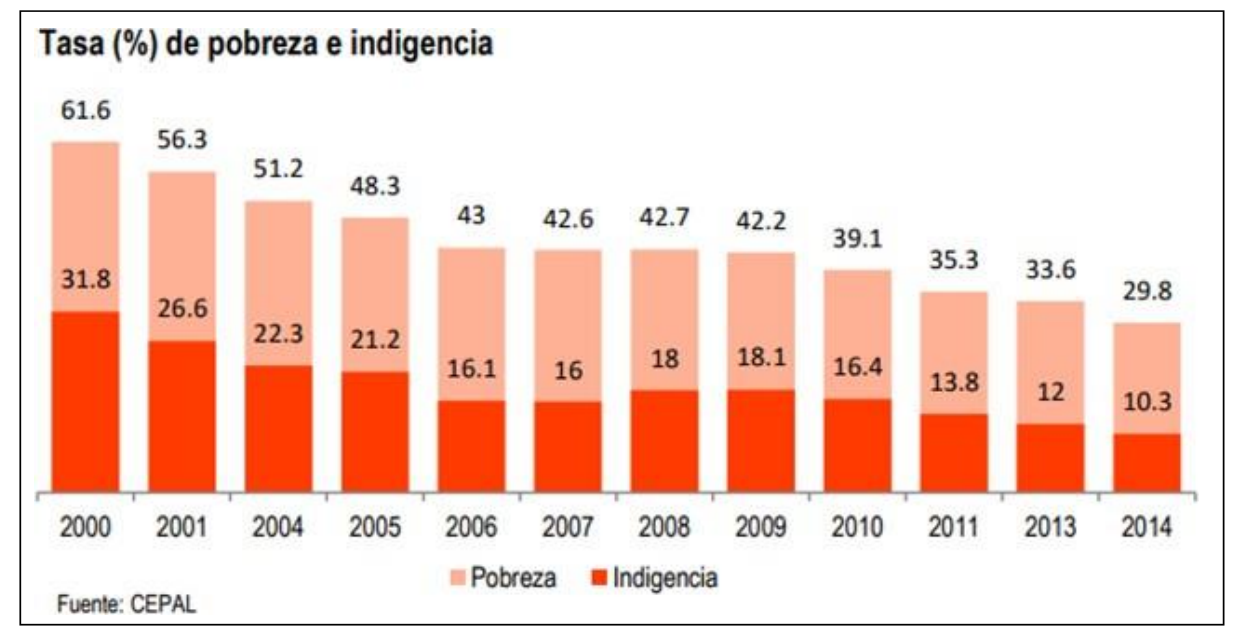

Según la información otorgada por la CEPAL en el Ecuador ha disminuido el porcentaje de pobreza e indigentes a $29.8 \%$ en el 2014 en comparación al año 2000. Si analizamos estos dos datos se diría que en el año 2000 hubo mayor indigencia y pobreza y a su vez mayor subnutrición el cual se pudo haber ocasionado por el cambio de moneda que se dio en aquella época.

La Utilización tiene relación con la higiene; saneamiento; calidad e inocuidad de los alimentos; información nutricional; y la forma en que el cuerpo aprovecha los nutrientes.

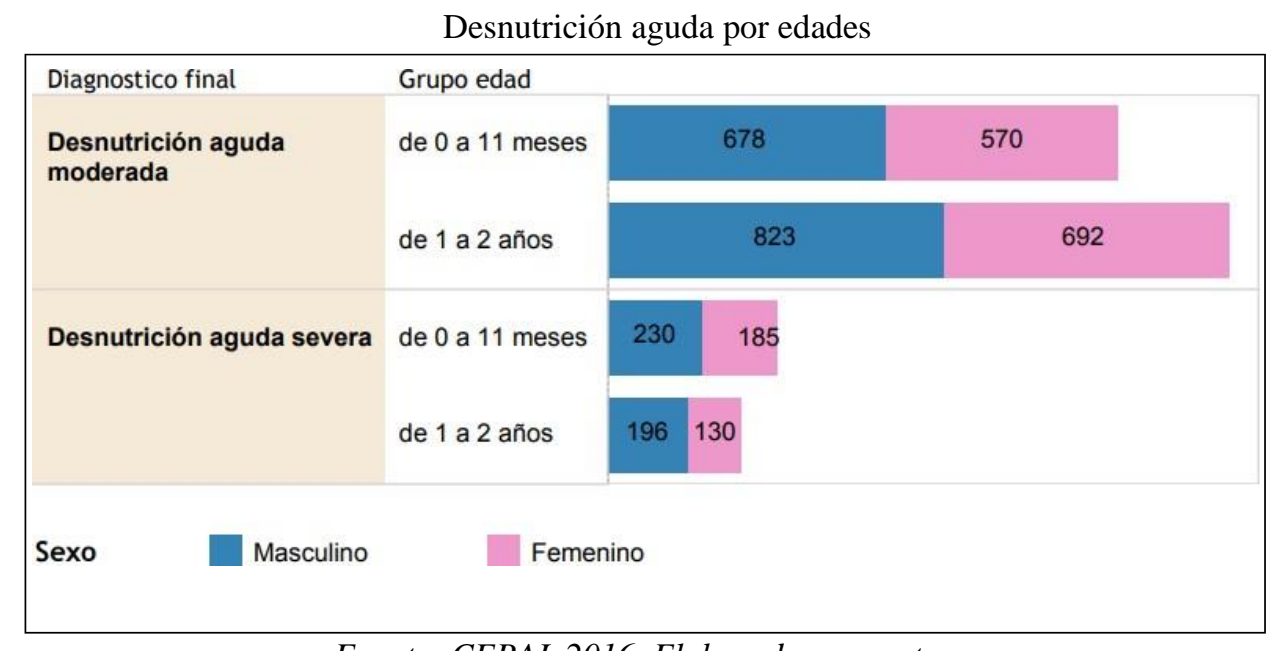

Fuente: CEPAL 2016, Elaborado por: autores

Para desnutrición aguda moderada el grupo más afectado es el de 1 a 2 años con el 54,83\% (1.515 casos) notificados; siendo en este grupo el sexo masculino el que presenta el 54.32\% (823 casos).

Para desnutrición aguda severa el grupo más afectado es el de 0 a 11 meses, con el 56\% (415 
casos) donde el 55,42\% (230 casos). 




Fuente: CEPAL 2016; Presentado por: autores

El acceso a las fuentes de agua también ha mejorado en los últimos años llegando a un porcentaje del $87 \%$.



Presentado por: autores

La Estabilidad hace relación con las capacidades de reacción ante imprevisto, crisis políticas, económicas, sociales y catástrofes naturales que puedan cambiar las condiciones de las otras dimensiones, generando riesgo y vulnerabilidad. 


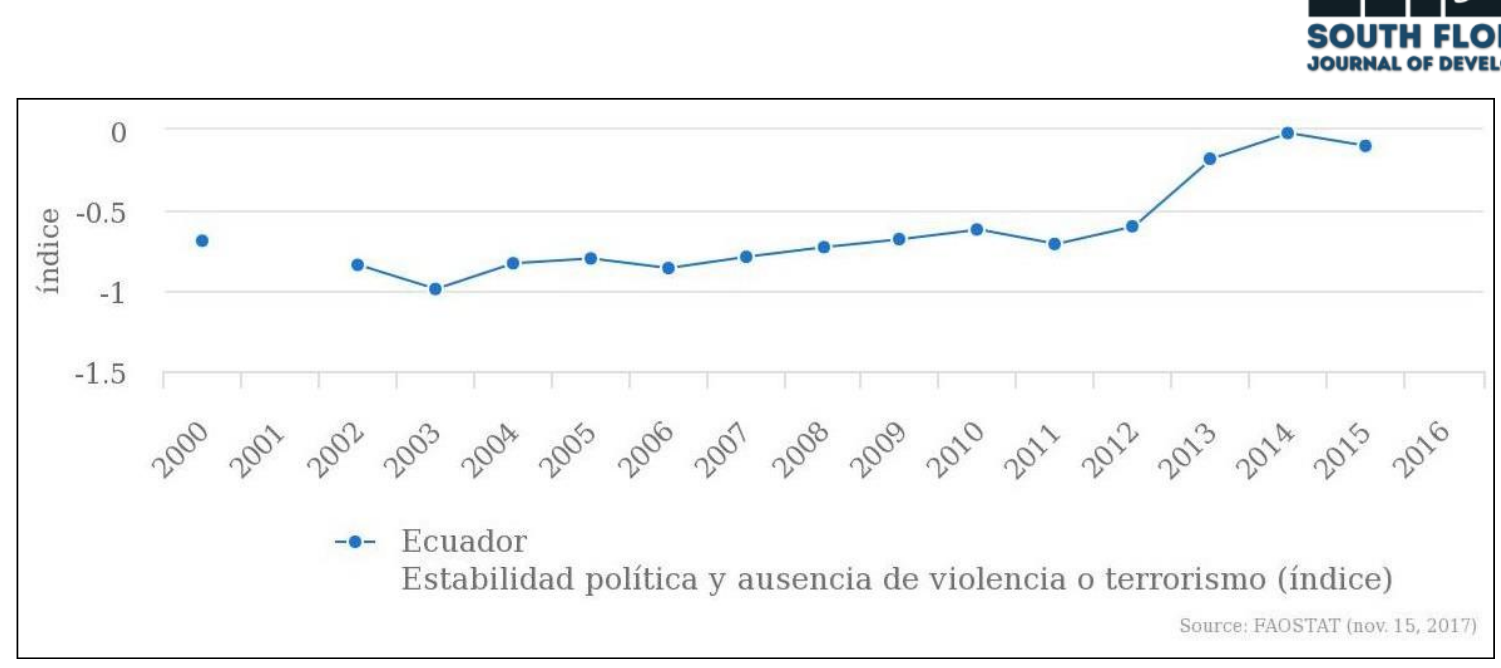

Presentado por: autores

En la siguiente ilustración se observa el valor de las importaciones de alimentos respecto a las exportaciones que en el año 2013 tuvo una decaída.



Presentado por: autores

\section{CONCLUSIÓN}

La Seguridad alimentaria en el Ecuador ha ido mejorando aunque todavía nos falta mucho camino por recorrer, de acuerdo con la investigación estamos haciendo lo mejor posible como intervenciones por medio de SAN las cuales son programas de alimentación escolar, estrategias nacionales para la igualdad y la erradicación de la pobreza, debemos seguir hacer proyectos que traten con la agricultura para que el nivel de personas que mueren por falta de alimentos (desnutrición), sea bajo esto ayuda a evitar que los alimentos tengan productos químicos para no perjudicar la salud, donde debemos tener una seguridad sumamente sana para poder hacer posible esta disminución de hambre se debe realizar campañas que trate sobre la seguridad alimenticia, para así poder tener acceso a los alimentos donde consumirlo puede ser más seguro gracias a las campañas que nos ayudaría a mejorar tanto en lo personal como en lo saludable . 


\section{BIBLIOGRAFÍA}

Asamblea Nacional. (2008). Constitución de la República del Ecuador. Manabí.

Elizabeth, R. I. M., Maribel, H. R. Z., Jesús, G. A., \& Manuel, M. R. V. INFLUENCIA DE LA RESPONSABILIDAD SOCIAL EN LA DECISIÓN DE COMPRA EN CONSUMIDORES DE CIUDAD VICTORIA. (2021)

FAO. (1996). Cumbre Mundial sobre la Alimentación. En Artículo 1.

FAO. (2009). SEGURIDAD ALIMENTARIA Y NUTRICIONAL EN EL ECUADOR.

Ley Orgánica del Régimen de la Soberanía Alimentaria. (2009). Obtenido de http://www.soberaniaalimentaria.gob.ec/prueba/servicios/wp-

content/uploads/downloads/2016/05/LORSA.pdf

Plataforma CELAC. (2015). Obtenido de http://plataformacelac.org/es/plan-celac Serrano, V. (2016). La desnutrición infantil, un mal que cuesta revertir. EL UNIVERSO. Sigifredo Briones Mejía. (1 de 12 de 2008). Seguridad Alimentaria. el diario.ec, pág. 1. 